\title{
Numerical Simulation of Sodium Droplet Combustion
}

\author{
Tadashi Watanabe
}

\begin{abstract}
The combustion of sodium droplet is simulated numerically. The flow fields in and around the droplet are obtained by solving the Navier-Stokes equations and the energy conservation equations. The reaction rate for the pre-ignition stage combustion is modeled using a kinetics based model, while that for the vapor phase after ignition is based on the mass transfer rate of oxygen. The droplet motion with temperature variation is shown, and compared with the existing experimental data, Reasonable agreement is shown between the simulated and the experimental results.
\end{abstract}

Keywords—sodium droplet, combustion, numerical simulation

\section{Introduction}

Sodium leakage is one of the important phenomena during the core disruptive accident of sodium cooled fast breeder reactors. Sodium is highly reactive, and the spray fire including droplets are significant because of large interfacial area for combustion. Experiments have been performed for a single droplet combustion by the institute relating to the development of fast reactors [1,2]. Burning rates and velocities of falling sodium droplets were measured. The combustion of suspended sodium droplet has been observed experimentally $[3,4]$, and the temperature variation in the burning droplet was shown. Numerical simulations have also been performed for the falling droplet [5] and for the spray combustion [6]. The detailed combustion phenomena in the suspended droplet was, however, not discussed yet.

In this study, the combustion of suspended sodium droplet [4] is simulated numerically. The increase in droplet temperature during the pre-ignition stage and the postignition stage is compared with the experimental observation, and the flow field including the droplet motion is discussed.

\section{Numerical Simulation}

\section{A. Combustion model}

The surface oxidation reaction occurs when the sodium droplet with relatively low temperature is released in atmospheric air. The droplet temperature increases due to this reaction heat on the droplet surface during the preignition stage, and the combustion in vapor phase occurs

\section{Tadashi Watanabe}

Research Institute of Nuclear Engineering, University of Fukui Japan after ignition due to the evaporation when the droplet temperature becomes sufficiently high.

Two chemical reactions occur mainly during the sodium combustion.

$$
\begin{aligned}
& 4 \mathrm{Na}+\mathrm{O}_{2} \rightarrow 2 \mathrm{Na}_{2} \mathrm{O} \\
& 2 \mathrm{Na}+\mathrm{O}_{2} \rightarrow \mathrm{Na}_{2} \mathrm{O}_{2}
\end{aligned}
$$

Sodium peroxide in (2) is not stable, and decomposes into sodium monoxide above 950K [6]. Equation (1) for the sodium monoxide is thus considered in this study.

The surface reaction rate for relatively low temperature conditions before ignition is modeled using the kinetics model [7].

$$
r=v W_{N a}\left(\rho_{g} Y_{O} / W_{O}\right)^{n} B_{s} \exp [-E /(R T)],
$$

where $\mathrm{r}$ is the reaction rate, $v$ the stoichiometric coefficient, $\rho$ the density, $\mathrm{W}$ the molecular weight, $\mathrm{Y}$ the mole fraction, $\mathrm{n}$ the reaction order, Bs the frequency factor of the surface reaction, $\mathrm{E}$ the activation energy, $\mathrm{R}$ the universal gas constant, $\mathrm{T}$ the temperature and the subscripts $\mathrm{Na}, \mathrm{g}$ and $\mathrm{O}$ denote sodium, the ambient gas and oxygen, respectively. The frequency factor is $320 \mathrm{~m} / \mathrm{s}$ and $\mathrm{E} / \mathrm{R}$ is $6350 \mathrm{~K}$ according to the experiment [7].

The vapor phase combustion after ignition is modeled using the mass transfer rate of oxygen. The vapor reaction rate for high temperature conditions is given by [6]

$$
r=4 M_{O},
$$

where Mo indicates the mass transfer rate of oxygen from the ambient gas to the reaction zone. This equation corresponds to the reaction equation (1), and the reaction rate of sodium is four times larger than the reaction rate of oxygen. The mass transfer rate is calculated using the mole fraction of oxygen.

$$
M_{O}=K_{c}\left(\rho_{g} / W_{O}\right)\left(Y_{\mathrm{O}}-Y_{O f}\right),
$$

where $\mathrm{Kc}$ is the mass transfer coefficient and $\mathrm{Y}_{\mathrm{Of}}$ is the mole fraction of oxygen at the reaction zone, which is calculated by taking into account the saturation pressure of sodium vapor. The mass transfer coefficient is evaluated using the following equation based on the flow field.

$$
K_{c}=(D / d)\left(2.0+0.6 \operatorname{Re}^{0.5} S c^{0.33}\right)
$$

where $\mathrm{D}$ is the molecular diffusivity and $\mathrm{d}$ is the droplet diameter, Re and $\mathrm{Sc}$ are, respectively, the Reynolds number and the Schmidt number defined by the following equations.

$$
\operatorname{Re}=\left(\rho_{g} u d / \mu_{g}\right)
$$




$$
S c=\left[\mu_{g} /\left(\rho_{g} D\right)\right]
$$

where $\mu_{\mathrm{g}}$ is the viscosity of the ambient gas.

\section{B. Flow field calculation}

The flow field including the burning droplet is simulated using the computational fluid dynamics (CFD) software Ansys Fluent [8]. The Navier Stokes equations together with the energy conservation equations are solved by the finite volume method in Fluent. The volume of fluid model is used to simulate the behavior of sodium droplet. The second order implicit method is used for time integration and the third order monotone upstream-centered scheme for conservation laws (MUSCL) is used for spatial discretization. The pressure-implicit with splitting of operators (PISO) method is used for calculations of pressure and velocity.

The mass transfer coefficient given by (6) and the mole fraction of oxygen at the reaction zone in (5) are temperature dependent, and evaluated by interpolation of pre-determined values for several representative temperature points. The reaction rate is thus a function of droplet temperature. The source term in the energy conservation equation is calculated using the local temperature in and around the droplet. The temperature derivative of the reaction rate is also evaluated and implemented into the code using the user defined function.

\section{Simulation conditions}

A single sodium droplet suspended in flowing air is simulated in this study. The simulation conditions are almost the same as that used in the experiment [4]. The sodium droplet is suspended at the lower end of the verticallyoriented thin tube through which liquid sodium is pushed out. The direction of flowing air is upward. The droplet diameter is $4.0 \mathrm{~mm}$ and the initial temperature is $673 \mathrm{~K}$. The temperature and the velocity of flowing air are $303 \mathrm{~K}$ and $0.4 \mathrm{~m} / \mathrm{s}$, respectively.

The simulation region is a two-dimensional square region with a size of $12 \mathrm{~mm} \times 12 \mathrm{~mm}$. The simulation cell size is $0.2 \mathrm{~mm} \times 0.2 \mathrm{~mm}$ and the number of mesh cells is thus $60 \times 60$. The vertical tube for suspension of droplet is also modeled. The simulation region and the initial condition are shown in Fig. 1. The high temperature and the high density regions are shown in red color and the low temperature and the low density regions are in blue color in Fig. 1.

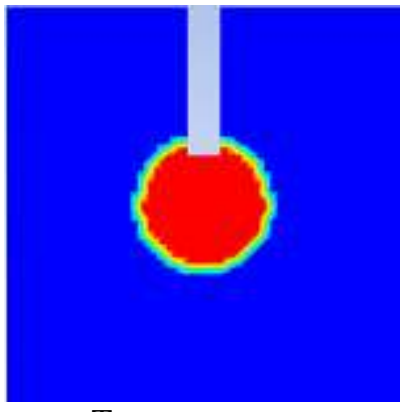

Temperature

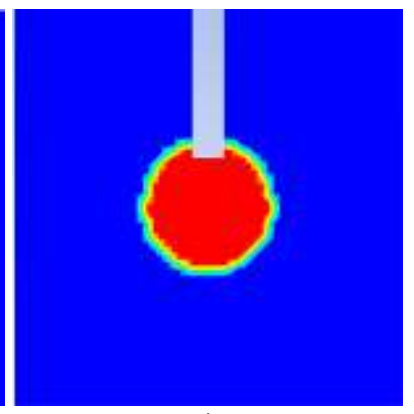

Density
Figure 1. Initial condition of suspended droplet.
The above described combustion model is implemented into the code using the user defined function as a source term in the energy conservation equation. The energy source term is calculated by using the reaction heat.

$$
S=q r \Delta s,
$$

where $\mathrm{S}$ is the source term in the energy equation, $\mathrm{q}$ the reaction heat, and $\Delta \mathrm{s}$ the reaction area in the calculation cell. The reaction heat of $634 \mathrm{~kJ} / \mathrm{mol}$ [1] is used in the following. The reaction area in the calculation cell is obtained as a function of volume fraction.

$$
\Delta s=\pi d^{2} \alpha(1-\alpha) \text {, }
$$

where 1 is the cell size and $\alpha$ is the volume fraction of gas or void fraction [9]. The reaction area is zero in the droplet region with the gas volume fraction of zero and in the pure gas region with the gas volume fraction of unity. The cell size is given by

$V=(\pi / 6) l^{3}$,

where $\mathrm{V}$ is the volume of the calculation cell. The evaluation method for the two-phase interfacial area using (10) and (11) are very simple and effective especially in the framework of CFD code, since the interfacial area is not defined in the calculation cell.

The interface of two-phase flow is generally not clear in CFD codes, especially in the volume of fluid model. The volume fraction of gas is unity in the pure gas phase and zero in the pure liquid phase. The volume fraction is, however, gradually varied across the two-phase interface over several calculation cells. The interface thus has a thickness in the numerical simulation, and fluid properties such as the viscosity and the density in the interfacial region are interpolated according to the smoothly varying volume fraction. The location with the volume fraction of 0.5 is usually regarded as the interface location. The pre-ignition combustion region on the droplet surface is thus defined as the region with the gas volume fraction from 0.4 to 0.5 , while the vapor phase combustion region is defined from 0.65 to 0.7 in our simulations.

\section{Results and Discussion}

The time history of the droplet temperature is shown in Fig. 2, along with the experimental data. It is shown that the droplet temperature increases from the initial temperature due to the pre-ignition combustion. The increase rate is relatively small both in the simulation and in the experiment up to $0.5 \mathrm{~s}$. The increase rate becomes large after $0.5 \mathrm{~s}$, and the droplet temperature reaches $850 \mathrm{~K}$ at about $1.0 \mathrm{~s}$. It is reported in the experiment that the ignition is observed at around the droplet temperature of $850 \mathrm{~K}$. The temperature history obtained by the present model up to $850 \mathrm{~K}$ is in good agreement with the experimental data. The combustion model for the pre-ignition stage is thus found to be reasonable.

The increase rate of droplet temperature becomes small after ignition in the experiment, and the experimental droplet temperature reaches its maximum value as shown in Fig. 2. The simulated droplet temperature, however, continue to increase, though the increase rate becomes small after ignition. The maximum temperature is about $970 \mathrm{~K}$ in 


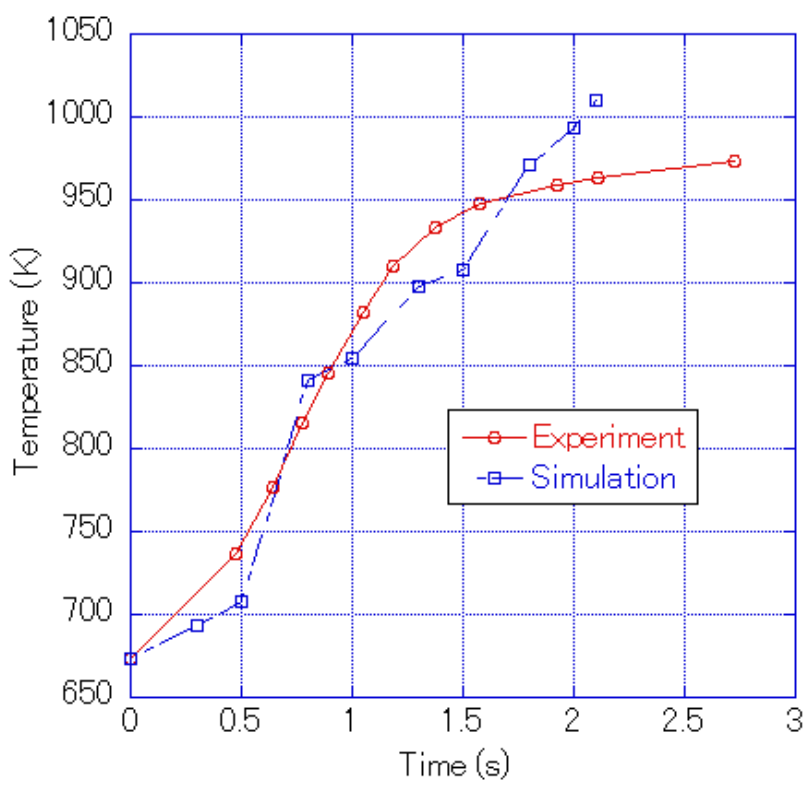

Figure 2. Increase in droplet temperature.

the experiment, while the evaporation temperature of sodium is about $1155 \mathrm{~K}$. It is thus reported in the experiment that there might have been some reasons for relatively low maximum temperature in the experiment [4]: the heat loss through the support tube for the droplet and the thermocouple itself. The heat conduction or heat capacity of the support tube is not simulated in this study, and the thermocouple is not modeled. The evaluation of these structural effects on the maximum droplet temperature would be desirable.

The flow fields in and around the droplet such as the temperature and density distributions are shown in Figs. 3 and 4 at $1.9 \mathrm{~s}$ and $2.0 \mathrm{~s}$, respectively. The maximum droplet temperature is about $980 \mathrm{~K}$, in these figures and much higher than the experimental result. This is because the reaction rate is still not so small at this temperature range in the simulation. The reaction rate in the combustion model used in this study becomes small due to the saturation pressure of the sodium vapor when the droplet temperature is very high and reaches the evaporation temperature. The effect of structure should thus be included in the simulation especially under high temperature conditions..

It is shown in Figs. 3 and 4 that the high temperature region spread widely from the droplet surface. Large

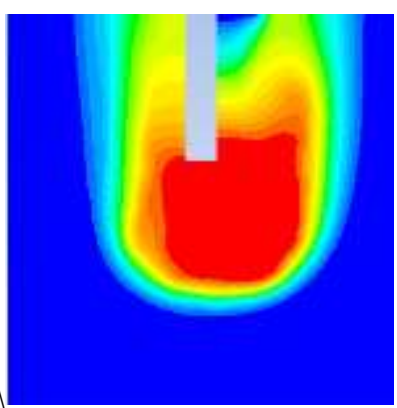

Temperature

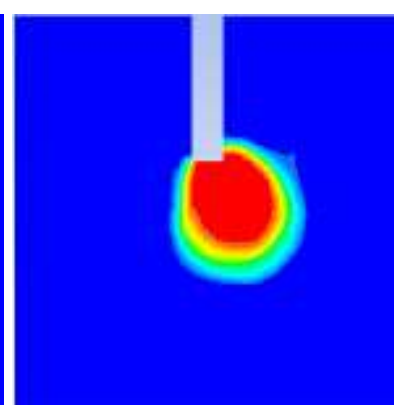

Density
Figure 3. Burning droplet at $1.9 \mathrm{~s}$

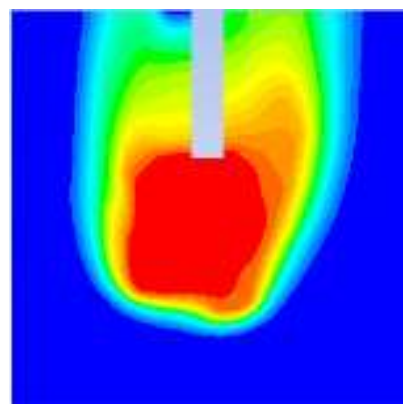

Temperature

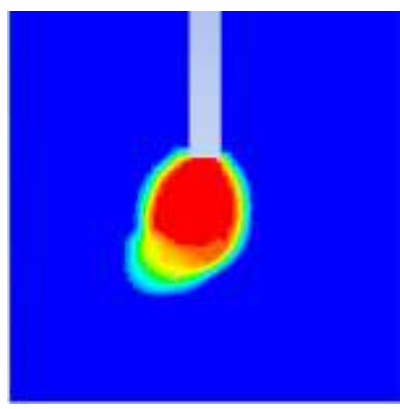

Density
Figure 4. Burning droplet at $2.0 \mathrm{~s}$.

temperature distribution is found around the droplet, since the inlet air temperature is $303 \mathrm{~K}$. Although the temperature distribution is rather uniform in the droplet, some tens of temperature difference is found in the droplet. The lowest position of the droplet, which is almost the stagnant location, shows the highest temperature.

Swinging motions and deformation are found in density distribution shown in Figs. 3 and 4. The temperature distribution seems to correspond to the density, and the high temperature region is also swinging. The red part of the density distribution indicates the pure droplet region and the blue part indicates the pure gas region. The green color shows the interpolated region and the reaction heat is added as the source term in this region. The location of source term is thus found to be swinging according to the flow field. The sodium droplet is treated as the solid sphere in the previous analyses [2,5], and the swinging motion and deformation cannot be simulated. The flow field in and around the droplet is simulated in this study, and it is found that the present model with the CFD approach is very much effective.

Examples of burning droplet observed in the experiment are shown in Fig. 5. Flow and temperature conditions in this figure are not the same as that in the simulation, but the combustion behavior is almost the same for other conditions in the experiment [4]. The burning sureface or the reaction zone is shown in the burning state picture in Fig. 5, since large reaction heat is generated from the reaction zone. The initial state almost corresponds to the droplet surface, and the reaction zone is found to be much larger than the droplet itself. The spread of high temperature region shown in Fig. 5 corresponds to the temperature distribution shown in Figs. 3 and 4 , and the present model with the CFD approach is again faound to be effective.

\section{Summary}

The combustion of suspended sodium droplet has been simulated numerically in this study. The flow fields in and around the droplet were obtained by solving the NavierStokes equations and the energy conservation equations in the framework of CFD code. The combustion model consisted of two models: one was for the pre-ignition stage and the other for the post-ignition stage. The reaction rate for the pre-ignition stage combustion was modeled using a kinetics based model, while that for the vapor phase after ignition was based on the mass transfer rate of oxygen. The reaction heat was included into the code as the source term. 


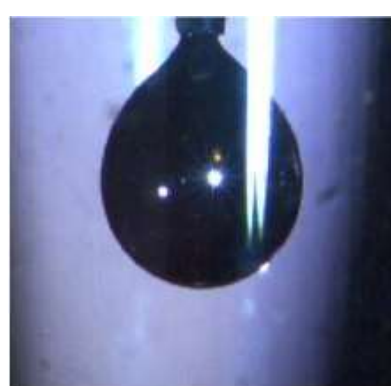

Initial state

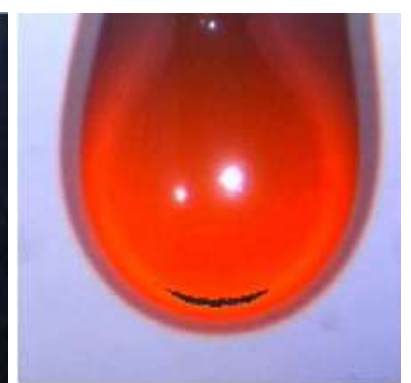

Burning state

Figure 5. Examples in experiment [4].

The temperature variation of burning droplet was shown, and compared with the existing experimental data, Reasonable agreement was obtained between the simulated and the experimental results for both the pre-ignition stage and the post-ignition stage.. The swinging motion of burning droplet and the spread of high temperature region were also shown.

\section{References}

[1] N. Doda, S. Ohno, and S. Miyahara, "Falling sodium droplet experiments (FD2)," JNC TN9400 2003-011, 2003,

[2] N. Doda, S. Ohno, and S. Miyahara, "Falling sodium droplet experiments (FD3), "JNC TN9400 2005-048, 2005.

[3] K. Sato, "Study on combustion behavior of sodium droplet," JNC TY9400 2003-008, 2003.

[4] K. Sato, "A experimental study on suspended sodium droplet combustion," JNC TY9400 2004-003, 2004

[5] Y. Okano and A. Yamaguchi, "Numerical simulation of a free falling liquid sodium droplet combustion," Annals Nucl. Eergy, vol. 30, pp. 1863-1878, 2003.

[6] S. M. Saravanan, P. M. Rao, B. K. Nashine, P. selvaraj, and P. Chellapandi, "NAFCON-SF: A sodium spray fire code for evaluating thermal consequences in SFR containment," Annals Nucl. Energy, vol. 90, pp. 389-409, 2016.

[7] A. Makino, "Ignition delay and limit of ignitability of a single sodium droplet: theor and experimental comparisons," Combustion and Flame, vol 134, pp. 149-152, 2003.

[8] ANSYS FLUENT user guide, Ansys Inc., 2006.

[9] J. Cai and T. Watanabe, "Numerical simulation of thermal stratification in cold legs by using OpenFOAM," Prog. Nucl. Sci. Tech., vol. 2, pp. 107-113, 2011.

Tadashi Watanabe

Ph. D. degree in Nuclear Engineering at Tokyo Institute of Technology, Japan, in 1985 .

Research engineer in the reactor safety division of Japan Atomic Energy Agency since 1985, and Professor in the research institute of nuclear engineering, University of Fukui, since 2012. The major research fields are nuclear reactor thermal hydraulics, reactor safety analysis, numerical simulations of two-phase flows, and computational science.

Member of Japan Atomic Energy Society, and Japan Mechanical Engineering Society. 